\title{
Reposição volêmica com hidroxietilamido ou solução de ringer lactato em cães com gastroenterite hemorrágica por parvovírus
}

\section{Volume replacement with hydroxyethyl starch or Ringer lactate solution in hemorrhagic gastroenteritis dogs with parvovirus}

\author{
Eduardo Ghiggi'; Vanessa Sasso Padilha²; Aury Nunes de Moraes ${ }^{3}$; \\ Marcos Paulo Antunes Lima'; Martielo Ivan Gehrcke²; \\ Rafael Messias Luiz ${ }^{5}$; Nilson Oleskovicz ${ }^{6}$
}

\begin{abstract}
Resumo
A parvovirose é uma doença infecciosa viral, altamente contagiosa, que acomete cães causando enterite aguda. A desidratação é um problema secundário e de evolução aguda, sendo este o foco principal do tratamento. O objetivo deste estudo foi avaliar dois tratamentos de reposição volêmica em cães com diagnóstico confirmado de parvovirose por detecção do antígeno nas fezes por meio de ELISA. Foram utilizados 15 cães, mestiços, machos e fêmeas, com idade de 3 a 6 meses, destes, quatro vieram a óbito durante o tratamento e 11 foram incluídos no estudo. Os pacientes foram alocados aleatoriamente em dois grupos: Grupo Hidroxietilamido 130/0,4 (HES, n=6) e Grupo Ringer Lactato (RL, n=5), os quais receberam fluidoterapia com hidroxietilamido 130/0,4 e solução de Ringer Lactato, respectivamente, com base nos cálculos de déficit hídrico. Todos os animais receberam por via intravenosa: cefalotina, metronidazol, ranitidina e metoclopramida. Durante o internamento, a cada 12 horas foram avaliados os gases sanguíneos arteriais, eletrólitos e realizou-se exame clínico completo (frequência cardíaca, frequência respiratória, temperatura retal, tempo de preenchimento capilar, vômito, apetite, desidratação, ganho de peso, hematoquezia, melena, hematêmese e dor abdominal) e a cada 24 horas hemograma. Em comparação ao basal, no grupo RL houve aumento nos valores de $\mathrm{PaO}_{2}$ e diminuição nos níveis de hemoglobina, hematócrito, fósforo e magnésio; e no grupo HES, ocorreu diminuição na porcentagem de desidratação, hemoglobina, fósforo e potássio. Não foram observadas diferenças significativas nos demais parâmetros entre os grupos. Ambos os tratamentos foram efetivos na reposição volêmica dos pacientes, não diferindo em nenhuma variável analisada.
\end{abstract}

Palavras-chave: Parvovírus, Eletrólitos, Hidroxietilamido 130/0,4, cães

\footnotetext{
Abstract

Parvovirus is an infectious viral disease, highly contagious, affecting dogs causing acute enteritis. Dehydration is a minor problem and acute evolution, which is the main focus of treatment. The objective of this study was to evaluate two treatments volume replacement in dogs with confirmed diagnosis of

${ }^{1}$ Médico Veterinário Autônomo, Lages, SC. E-mail: eduardo.ghiggi@gmail.com

${ }^{2}$ Discente(s) de Doutorado(s), Centro de Ciências Agroveterinárias/Universidade do Estado de Santa Catarina, UFCG, Lages, SC. E-mail: vanessa.sasso@hotmail.com; martielogehrcke@hotmail.com

${ }^{3}$ Prof. do Centro de Ciências Agroveterinárias, UFCG, Lages, SC. E-mail: a2anm@cav.udesc.br

${ }^{4}$ Discente de Mestrado, Centro de Ciências Agroveterinárias, UFCG, Lages, SC. E-mail: marcos_paulo@hotmail.com

${ }^{5}$ Médico Veterinário Anestesista, Universidade Federal do Paraná, UFPR, Campus Palotina, PR. E-mail: rafaelmessiasluiz@,

${ }^{6}$ Orientador, Prof. de Anestesiologia Veterinária, Centro de Ciências Agroveterinárias, UFCG, Lages, SC. E-mail: a2no@cav.
} gmail.com udesc.br

* Autor para correspondência 
parvovirus infection by antigen detection in stool by ELISA. A total of $15 \mathrm{dogs}$, mixed, male and female, aged from 3 to 6 months, these four they had died during treatment and 11 were included in the study. Patients were randomly divided into two groups: hydroxyethyl starch 130/0.4 (HES, n=6) and Group Ringer Lactate (RL, n=5), which received fluid resuscitation with hydroxyethyl starch 130/0.4 and Ringer's solution lactate, respectively, based on calculations of fluid deficit. All animals received intravenously: cephalothin, metronidazole, ranitidine and metoclopramide. During hospitalization, every 12 hours were evaluated arterial blood gas, electrolyte dosing and clinical examination (heart rate, respiratory rate, rectal temperature, capillary refill time, vomiting, appetite, dehydration, weight gain, hematochezia, melena, hematemesis and presence abdominal pain) and 24 hours each blood count was performed. In group RL was increase in the values of $\mathrm{PaO}_{2}$ and decreases in hemoglobin, hematocrit, phosphorus and magnesium, and group HES, a decrease in the percentage of dehydration, hemoglobin, phosphorus and potassium. There were no differences in other parameters between the groups. Both treatments were effective in fluid replacement of patients, no difference in any variable analyzed.

Key words: Parvovirus, electrolytes, hydroxyethyl starch 130/0.4, dogs

\section{Introdução}

O parvovírus canino (CPV) é de elevada morbidade e mortalidade em filhotes com menos de seis meses de idade, especialmente os não vacinados (McCAW; HOSKINS, 2006). A sobrevivência de filhotes infectados varia de $9 \%$ para os não tratados, e mais de $90 \%$ para aqueles tratados corretamente dentro de hospitais e clínicas veterinária (OTTO et al., 2001). Parasitos, protozoários, bactérias, vírus, agentes estressores como o desmame e condições de superlotação, e a insuficiente imunidade passiva ou ativa podem contribuir para o desenvolvimento e gravidade da doença (SAVIGNY; MACINTIRE, 2007).

O diagnóstico clínico de infecção por CPV não é confiável, uma vez que vários outros agentes podem causar diarréia em cães. Assim o diagnóstico clínico deve ser sempre confirmado por testes laboratoriais, tais como microscopia eletrônica, isolamento viral, hemaglutinação fecal, aglutinação em látex, imunoeletroforese, imunocromatografia e PCR (reação de polimerização em cadeia) (ESFANDIARI; KLINGEBORN, 2000).

A fluidoterapia intravenosa comumente é indicada e deve ser mantida até que o vômito cesse e a ingestão de água seja normalizada (HOSKINS, 2004). Porém nos casos não reativos a fluidoterapia convencional, recomenda-se a utilização de colóides e a adição de glicose para manter a glicemia entre 120 e 160 mg/dl. A manutenção ou mesmo a restauração da volemia com soluções cristalóides isotônicas, como é o caso da solução de Ringer Lactato, requer volumes de infusão aproximadamente duas a seis vezes superiores aos das soluções colóides para se alcançar o mesmo resultado clínico (SHERDING, 2003).

Por outro lado, o hidroxietilamido (HES 130/0,4) apresenta uma capacidade de expansão de aproximadamente $100 \%$, ou seja, aumenta o volume plasmático na mesma proporção do volume infundido por 4 a 6 horas (WALTZINGER; BEPPERLING; PABST, 1999). Ainda, segundo LV et al. (2006), os colóides apresentam efeitos antiinflamatórios, sendo úteis no tratamento de enfermidades onde há quebra de barreira, como no caso da gastroenterite hemorrágica, no entanto, alguns autores afirmam que estudos são necessários para confirmar esta teoria (MARX et al., 2006). Desta forma, o objetivo deste estudo foi avaliar as alterações clínicas, hematológicas, hemogasométricas, o tempo de recuperação e a taxa de mortalidade de cães com diagnóstico confirmado de parvovirose, tratados com hidroxietilamido 130/0,4 ou solução de Ringer lactato.

\section{Material e Métodos}

Foram utilizados 15 cães, mestiços, machos e fêmeas, com idade entre três e seis meses com diagnóstico definitivo de gastroenterite hemorrágica 
por parvovírus, provenientes da rotina do Hospital de Clínicas Veterinárias "Lauro Ribas Zimmer" (HCV/CAV/UDESC). Destes, quatro vieram a óbito nas primeiras horas após o internamento, e 11 foram incluídos no estudo. Durante o período de tratamento, os pacientes foram mantidos em isolamento, em gaiolas individuais, sendo incluídos no estudo somente após autorização dos proprietários.

A confirmação do diagnóstico foi realizada com kit de teste para detecção do antígeno do parvovírus nas fezes dos animais (Antigen Rapid CPV Ag Test Kit $\left.{ }^{\circledR}\right)$. A partir deste momento, os animais foram alocados aleatoriamente em dois grupos: Grupo Hidroxietilamido 130/0,4 (HES, $n=6$ ) os quais receberam fluidoterapia com hidroxietilamido 130/0,4 (peso molecular 130.000 Dalton e substituição molar de 0,4 , em solução de ringer acetato) e Grupo Ringer Lactato (RL, $\mathrm{n}=5$ ) os quais receberam tratamento com solução de Ringer Lactato. Após a confirmação do diagnóstico e precedendo qualquer tratamento (M0) avaliouse: peso, grau de desidratação (\%), presença de dor abdominal, apetite, presença de sangue vivo nas fezes (hematoquezia), presença de sangue digerido nas fezes (melena), êmese ou hematêmese, bem como, frequência cardíaca (FC), frequência respiratória $(f)$, temperatura retal (TR) e tempo de preenchimento capilar (TPC). Após, as avaliações eram realizadas a cada 12 horas, sendo M1, M2, M3, M4 e M5 correspondente a 12, 24, 36, 48 e 60 horas após início do tratamento, respectivamente.

Logo após, realizou-se a tricotomia da região das veias jugulares e cefálicas para realização de coleta de sangue e cateterização venosa, respectivamente. Para realização da cateterização venosa, após a tricotomia, foi realizado anti-sepsia com álcool $70^{\circ}$ e solução de iodo-povidona, seguidos de punção, introdução e fixação do cateter e bandagem. Os cateteres venosos eram verificados durante as avaliações para se detectar a presença de flebite e a sua substituição era realizada a cada 72 horas ou quando o cateter estava inviável para administração da fluidoterapia. O sangue venoso era coletado uma vez ao dia para realização de hemograma e dosagem sérica total de eletrólitos (cálcio, sódio, potássio, magnésio, fósforo e cloretos). Também realizou-se tricotomia das regiões femoral e tibialdorsal, para coleta de sangue arterial, duas vezes ao dia, realizada por punção com seringa de $1 \mathrm{ml}$ heparinizada, para análise hemogasométrica (pressão parcial de oxigênio $\left(\mathrm{PaO}_{2}\right)$, pressão parcial de dióxido de carbono $\left(\mathrm{PaCO}_{2}\right)$, potencial de hidrogênio $(\mathrm{pH})$, bicarbonato $\left(\mathrm{HCO}_{3}^{-}\right)$, déficit de base (DB) e saturação de oxihemoglobina $\left(\mathrm{SaO}_{2}\right)$ ).

Para a realização da fluidoterapia, avaliavamse os parâmetros clínicos do paciente, juntamente com a avaliação hematológica, hemogasométrica e estimativa do grau de desidratação sendo considerado: 5\% (mucosas secas), 6-8\% (redução da elasticidade cutânea), $8-10 \%$ (hipoperfusão com tempo de preenchimento capilar (TPC) $>3$ segundos), 10-12\% (hipotensão) e acima de 12\% (choque).

Determinado o grau de desidratação calculavase a fluidoterapia necessária para cada paciente em 24 horas, considerando-se as perdas diárias, de manutenção e de reposição volêmica, sendo a manutenção (Peso $(\mathrm{Kg})$ x 40$)$, de reposição volêmica (Peso $(\mathrm{Kg})$ x \% de desidratação x 10). As perdas diárias no HES eram calculadas pela seguinte formula: $30 \mathrm{ml} / \mathrm{kg} /$ dia de hidroxietilamido 130/0,4, divididos em duas administrações por um período de 30 minutos e no RL, peso $(\mathrm{Kg}) \times 10$ x K, sendo K: 40 para diarréia, 50 para vômito e 60 para diarréia e vômito.

Após ajuste da fluidoterapia em cada grupo, adminstrava-se cefalotina $30 \mathrm{mg} / \mathrm{kg}$ IV (cada 8 horas), metronidazol $25 \mathrm{mg} / \mathrm{kg}$ IV (cada 12 horas), metoclopramida $0,5 \mathrm{mg} / \mathrm{kg}$ IV (cada 8 horas) e ranitidina 2,2mg/kg IV (cada 12 horas). Todos os animais foram submetidos à restrição hídrica e alimentar durante o período que estivessem apresentando vômito, tendo o retorno gradual a ingestão hídrica e alimentar (ração Intestinal - Royal 
Canin $\left({ }^{\circledR}\right)$ com o cessar do sinal clínico de vômito. Não foi fornecido nenhum suporte energético para os animais, sendo que estes recebiam alta clínica quando não apresentassem mais sinais de gastroenterite como a diarréia e o vômito e restabelecimento do apetite, sendo o tratamento com antibiótico mantido por um período mínimo de 10 dias.

Os dados quantitativos dentro dos grupos, entre tempos, foram submetidos à análise de variância de uma via com repetições múltiplas (ANOVA RM) seguida pelo teste de Student Newmann Keuls, e teste $t$ entre os grupos, dentro do mesmo tempo $\mathrm{P}>0,05$.

\section{Resultados e Discussão}

Dos 15 cães submetidos ao estudo, quatro vieram a óbito, sendo que destes, apenas dois sobreviveram até 36 horas após o internamento. Devido ao curto período de avaliação, estes pacientes foram excluídos da análise estatística.

Não houve diferença significativa entre os grupos para os parâmetros: $\mathrm{FC}, f$, TR, grau de desidratação, tempo de preenchimento capilar, apetite, pulso, vômito, diarréia, melena, hematoquezia, dor abdominal, ganho de peso, $\mathrm{pH}, \mathrm{PaO}_{2}, \mathrm{PaCO}_{2}$, íons $\mathrm{HCO}_{3}^{-}, \mathrm{SaO}_{2}, \mathrm{DB}$, íons sódio, magnésio, fósforo, potássio, cloro, cálcio, eritrócitos, hemoglobina, hematócrito, volume corpuscular médio, concentração de hemoglobina corpuscular média, leucócitos, neutrófilos, linfócitos, bastonetes, basófilos, eosinófilos e monócitos, em todos os momentos avaliados (Tabelas 1, 2 e 3). Bem como não foram observadas diferenças dentro do grupo para os valores de FC, $f$ e TR (Tabela 1) e também não houve diferença dentro do grupo para os parâmetros $\mathrm{pH}, \mathrm{PaCO}_{2}, \mathrm{HCO}_{3}, \mathrm{DB}, \mathrm{SaO}_{2}$ e Cálcio (Tabela 2) e todos os parâmetros hematológicos, exceto hemoglobina e hematócrito (Tabela 3 ).

Tabela 1. Valores médios e desvio padrão, dos parâmetros FC (freqüência cardíaca), $f$ (freqüência respiratória), TR (temperatura retal) e grau de desidratação (\%), nos grupos RL e HES, nos momentos $M_{0}$ até $M_{5}$.

\begin{tabular}{|c|c|c|c|c|c|c|c|}
\hline Parâmetros & Grupo & $\mathbf{M}_{0}$ & $\mathbf{M}_{1}$ & $\mathbf{M}_{2}$ & $\mathbf{M}_{3}$ & $\mathbf{M}_{4}$ & $\mathbf{M}_{5}$ \\
\hline \multirow{6}{*}{$\begin{array}{l}\text { FC } \\
\text { (bat/min) }\end{array}$} & \multirow{2}{*}{ HES } & 165 & 186 & 137 & 143 & 153 & 160 \\
\hline & & \pm 52 & \pm 45 & \pm 58 & \pm 56 & \pm 28 & \pm 48 \\
\hline & \multirow{2}{*}{ RL } & 142 & 157 & 147 & 137 & 151 & 142 \\
\hline & & \pm 36 & \pm 45 & \pm 58 & \pm 21 & \pm 51 & \pm 14 \\
\hline & \multirow{2}{*}{ HES } & 29 & 24 & 28 & 25 & 29 & 22 \\
\hline & & \pm 6 & \pm 3 & \pm 11 & \pm 6 & \pm 12 & \pm 6 \\
\hline \multirow{4}{*}{$\begin{array}{l}f \\
(\operatorname{mov} . / \mathrm{min})\end{array}$} & \multirow{2}{*}{ RL } & 33 & 31 & 25 & 25 & 23 & 24 \\
\hline & & \pm 10 & \pm 6 & \pm 7 & \pm 9 & \pm 2 & \pm 0 \\
\hline & \multirow{2}{*}{ HES } & 38,2 & 38,2 & 38,1 & 37,9 & 38,3 & 38,5 \\
\hline & & $\pm 0,3$ & $\pm 0,3$ & $\pm 0,1$ & $\pm 0,5$ & $\pm 0,3$ & $\pm 0,7$ \\
\hline \multirow{2}{*}{$\begin{array}{l}\text { TR } \\
\left({ }^{\circ} \mathrm{C}\right)\end{array}$} & \multirow{2}{*}{ RL } & 38,6 & 39,0 & 39,0 & 38 & 38,7 & 38,6 \\
\hline & & $\pm 0,37$ & $\pm 0,90$ & $\pm 0,38$ & $\pm 0,35$ & $\pm 0,23$ & $\pm 0,5$ \\
\hline \multirow{4}{*}{$\begin{array}{l}\text { Desidratação } \\
(\%)\end{array}$} & \multirow{2}{*}{ HES } & 7 & 6 & 2 & 2 & 2 & 2 \\
\hline & & $\pm 1 \mathrm{a}$ & $\pm 2 \mathrm{~b}$ & $\pm 3 \mathrm{~b}$ & $\pm 2 \mathrm{~b}$ & $\pm 2 \mathrm{~b}$ & $\pm 3 \mathrm{~b}$ \\
\hline & \multirow{2}{*}{$\mathbf{R L}$} & 6 & 6 & 4 & 4 & 1 & 2 \\
\hline & & \pm 1 & \pm 1 & \pm 2 & \pm 2 & \pm 2 & \pm 3 \\
\hline
\end{tabular}

a, $\mathrm{b}$ - Letras minúsculas diferentes entre os momentos dentro de cada grupo, indicam diferenças significativas (P<0,05). Momentos: M0 - basal; M1 - 12 horas; M2 - 24 horas; M3 - 36 horas; M4 - 48 horas; M5 - 60 horas.

Fonte: Elaboração dos autores. 
Tabela 2. Valores médios e desvio padrão, do Potencial hidrogeniônico $(\mathrm{pH})$, Pressão arterial de dióxido de carbono $\left(\mathrm{PaCO}_{2}\right)$, Pressão arterial de oxigênio $\left(\mathrm{PaO}_{2}\right.$, bicarbonato $\left(\mathrm{HCO}_{3}\right)$, déficit de base $(\mathrm{DB})$, saturação de oxihemoglobina $\left(\mathrm{SaO}_{2}\right)$ e dosagem de eletrólitos ( $\mathrm{Ca}, \mathrm{P}, \mathrm{Mg}$ e K) nos grupos RL e HES, nos momentos $\mathrm{M}_{0}, \mathrm{M}_{1}, \mathrm{M}_{2}, \mathrm{M}_{3}, \mathrm{M}_{4}$ e $\mathrm{M}_{5}$.

\begin{tabular}{|c|c|c|c|c|c|c|c|}
\hline & Grupo & $\mathbf{M}_{0}$ & $\mathbf{M}_{1}$ & $\mathbf{M}_{2}$ & $\mathbf{M}_{3}$ & $\mathbf{M}_{4}$ & $\mathbf{M}_{5}$ \\
\hline \multirow{3}{*}{ pH } & HES & $\begin{array}{c}7,37 \\
\pm 0,02\end{array}$ & $\begin{array}{c}7,37 \\
\pm 0,02\end{array}$ & $\begin{array}{c}7,38 \\
\pm 0,04\end{array}$ & $\begin{array}{c}7,40 \\
\pm 0,06\end{array}$ & $\begin{array}{c}7,39 \\
\pm 0,10\end{array}$ & $\begin{array}{c}7,43 \\
\pm 0,03\end{array}$ \\
\hline & RL & $\begin{array}{c}7,42 \\
\pm 0,03\end{array}$ & $\begin{array}{c}7,42 \\
\pm 0,03\end{array}$ & $\begin{array}{c}7,44 \\
\pm 0,03\end{array}$ & $\begin{array}{c}7,45 \\
\pm 0,004\end{array}$ & $\begin{array}{c}7,43 \\
\pm 0,04\end{array}$ & $\begin{array}{c}7,37 \\
\pm 0,07\end{array}$ \\
\hline & HES & $\begin{array}{l}34,2 \\
\pm 6,4\end{array}$ & $\begin{array}{l}33,9 \\
\pm 7,6\end{array}$ & $\begin{array}{l}31,8 \\
\pm 2,1\end{array}$ & $\begin{array}{c}35 \\
\pm 6,2\end{array}$ & $\begin{array}{l}31,4 \\
\pm 5,5\end{array}$ & $\begin{array}{l}33,3 \\
\pm 5,6\end{array}$ \\
\hline \multirow[t]{2}{*}{$\mathrm{PaCO}_{2}$} & RL & $\begin{array}{c}44,2 \\
\pm 18,9\end{array}$ & $\begin{array}{c}37,9 \\
\pm 20,7\end{array}$ & $\begin{array}{c}39,1 \\
\pm 17,9\end{array}$ & $\begin{array}{c}52,2 \\
\pm 18,7\end{array}$ & $\begin{array}{c}40,8 \\
\pm 22,3\end{array}$ & $\begin{array}{l}33,8 \\
\pm 1,1\end{array}$ \\
\hline & HES & $\begin{array}{c}65,3 \\
\pm 28,1\end{array}$ & $\begin{array}{c}60,7 \\
\pm 27,1\end{array}$ & $\begin{array}{c}83,6 \\
\pm 12,0\end{array}$ & $\begin{array}{c}58,2 \\
\pm 28,1\end{array}$ & $\begin{array}{c}75,6 \\
\pm 17,7\end{array}$ & $\begin{array}{c}75,3 \\
\pm 20,3\end{array}$ \\
\hline \multirow[t]{2}{*}{$\mathrm{PaO}_{2}$} & RL & $\begin{array}{c}70,8 \\
\pm 16,0 \mathrm{a}\end{array}$ & $\begin{array}{c}89,9 \\
\pm 10,0 \mathrm{~b}\end{array}$ & $\begin{array}{c}92,6 \\
\pm 17,3 \mathrm{~b}\end{array}$ & $\begin{array}{l}81,8 \\
\pm 4,7\end{array}$ & $\begin{array}{c}88,8 \\
\pm 10,8 \mathrm{~b}\end{array}$ & $\begin{array}{c}54,4 \\
\pm 29,4\end{array}$ \\
\hline & HES & $\begin{array}{l}19,4 \\
\pm 3,1\end{array}$ & $\begin{array}{l}19,4 \\
\pm 4,6\end{array}$ & $\begin{array}{l}18,8 \\
\pm 2,7\end{array}$ & $\begin{array}{l}21,3 \\
\pm 2,8\end{array}$ & $\begin{array}{l}19,2 \\
\pm 4,7\end{array}$ & $\begin{array}{l}21,9 \\
\pm 5,0\end{array}$ \\
\hline \multirow[t]{2}{*}{$\mathrm{HCO}_{3}$} & RL & $\begin{array}{l}20,5 \\
\pm 2,5\end{array}$ & $\begin{array}{l}18,7 \\
\pm 3,8\end{array}$ & $\begin{array}{l}19,7 \\
\pm 1,7\end{array}$ & $\begin{array}{l}21,1 \\
\pm 2,3\end{array}$ & $\begin{array}{l}18,8 \\
\pm 2,3\end{array}$ & $\begin{array}{l}20,3 \\
\pm 1,0\end{array}$ \\
\hline & HES & $\begin{array}{l}-4,8 \\
\pm 2,4\end{array}$ & $\begin{array}{l}-4,7 \\
\pm 3,8\end{array}$ & $\begin{array}{l}-5,0 \\
\pm 3,2\end{array}$ & $\begin{array}{l}-2,6 \\
\pm 2,9\end{array}$ & $\begin{array}{l}-4,4 \\
\pm 5,8\end{array}$ & $\begin{array}{l}-1,5 \\
\pm 4,6\end{array}$ \\
\hline \multirow[t]{2}{*}{ DB } & RL & $\begin{array}{l}-2,5 \\
\pm 2,7\end{array}$ & $\begin{array}{l}-4,4 \\
\pm 3,6\end{array}$ & $\begin{array}{l}-2,7 \\
\pm 1,4\end{array}$ & $\begin{array}{l}-2,1 \\
\pm 1,6\end{array}$ & $\begin{array}{l}-3,4 \\
\pm 2,1\end{array}$ & $\begin{array}{l}-3,5 \\
\pm 1,6\end{array}$ \\
\hline & HES & $\begin{array}{c}77,5 \\
\pm 29,2\end{array}$ & $\begin{array}{c}76,3 \\
\pm 22,9\end{array}$ & $\begin{array}{l}93,9 \\
\pm 3,4\end{array}$ & $\begin{array}{c}75,8 \\
\pm 25,1\end{array}$ & $\begin{array}{l}94,0 \\
\pm 4,6\end{array}$ & $\begin{array}{c}84,0 \\
\pm 24,3\end{array}$ \\
\hline \multirow[t]{2}{*}{$\mathrm{SaO}_{2}$} & RL & $\begin{array}{c}92,5 \\
\pm 6,37\end{array}$ & $\begin{array}{l}96,8 \\
\pm 1,0\end{array}$ & $\begin{array}{l}97,1 \\
\pm 1,4\end{array}$ & $\begin{array}{l}96,2 \\
\pm 0,6\end{array}$ & $\begin{array}{c}97,0 \\
\pm 0,82\end{array}$ & $\begin{array}{c}78,4 \\
\pm 23,7\end{array}$ \\
\hline & HES & $\begin{array}{c}9,1 \\
\pm 1,3\end{array}$ & $\begin{array}{c}7,8 \\
\pm 1,2\end{array}$ & $\begin{array}{c}8,0 \\
\pm 1,0\end{array}$ & $\begin{array}{c}9,1 \\
\pm 2,4\end{array}$ & $\begin{array}{c}8,2 \\
\pm 1,5\end{array}$ & $\begin{array}{c}7,8 \\
\pm 0,6\end{array}$ \\
\hline \multirow[t]{2}{*}{ Cálcio } & RL & $\begin{array}{c}9,8 \\
\pm 0,7\end{array}$ & $\begin{array}{c}9,0 \\
\pm 1,2\end{array}$ & $\begin{array}{c}8,2 \\
\pm 1,7\end{array}$ & $\begin{array}{c}7,9 \\
\pm 0,4\end{array}$ & $\begin{array}{c}8,5 \\
\pm 1,1\end{array}$ & $\begin{array}{c}7,5 \\
\pm 0,1\end{array}$ \\
\hline & HES & $\begin{array}{c}6,4 \\
\pm 0,7 \mathrm{a}\end{array}$ & $\begin{array}{c}4,5 \\
\pm 1,8 \mathrm{~b}\end{array}$ & $\begin{array}{c}5,0 \\
\pm 2,2 \mathrm{~b}\end{array}$ & $\begin{array}{c}4,8 \\
\pm 1,9 \mathrm{~b}\end{array}$ & $\begin{array}{c}4,4 \\
\pm 2,0 \mathrm{~b}\end{array}$ & $\begin{array}{c}4,5 \\
\pm 1,4 \mathrm{~b}\end{array}$ \\
\hline \multirow[t]{2}{*}{ Fósforo } & $\mathbf{R L}$ & $\begin{array}{c}6,93 \\
\pm 0,75 \mathrm{a}\end{array}$ & $\begin{array}{c}4,0 \\
\pm 1,4 \mathrm{~b}\end{array}$ & $\begin{array}{c}5,5 \\
\pm 3,0\end{array}$ & $\begin{array}{c}4,1 \\
\pm 1,0 \mathrm{~b}\end{array}$ & $\begin{array}{c}4,7 \\
\pm 0,6 \mathrm{~b}\end{array}$ & $\begin{array}{c}5,2 \\
\pm 0,1\end{array}$ \\
\hline & HES & $\begin{array}{c}1,6 \\
\pm 0,1\end{array}$ & $\begin{array}{c}1,8 \\
\pm 0,4\end{array}$ & $\begin{array}{c}1,9 \\
\pm 0,4\end{array}$ & $\begin{array}{c}2,3 \\
\pm 0,9\end{array}$ & $\begin{array}{c}2,3 \\
\pm 0,8\end{array}$ & $\begin{array}{c}2,3 \\
\pm 1,0\end{array}$ \\
\hline Magnésio & $\mathbf{R L}$ & $\begin{array}{c}2,3 \\
\pm 0,49 \mathrm{a}\end{array}$ & $\begin{array}{c}1,9 \\
\pm 0,1 \mathrm{~b}\end{array}$ & $\begin{array}{c}1,6 \\
\pm 0,1 \mathrm{~b}\end{array}$ & $\begin{array}{c}1,8 \\
\pm 0,3 \mathrm{~b}\end{array}$ & $\begin{array}{c}1,5 \\
\pm 0,2 \mathrm{~b}\end{array}$ & $\begin{array}{c}1,8 \\
\pm 0,2\end{array}$ \\
\hline \multirow[t]{2}{*}{ Potássio } & HES & $\begin{array}{c}4,4 \\
\pm 0,78 \mathrm{a}\end{array}$ & $\begin{array}{c}4,3 \\
\pm 0,3\end{array}$ & $\begin{array}{c}3,8 \\
\pm 0,5\end{array}$ & $\begin{array}{c}3,8 \\
\pm 0,7\end{array}$ & $\begin{array}{c}3,4 \\
\pm 0,7 \mathrm{~b}\end{array}$ & $\begin{array}{c}3,5 \\
\pm 0,7 \mathrm{~b}\end{array}$ \\
\hline & RL & $\begin{array}{c}4,6 \\
\pm 0,1 \\
\end{array}$ & $\begin{array}{c}4,0 \\
\pm 0,6 \\
\end{array}$ & $\begin{array}{c}4,0 \\
\pm 0,8 \\
\end{array}$ & $\begin{array}{c}4,1 \\
\pm 0,5 \\
\end{array}$ & $\begin{array}{c}4,0 \\
\pm 0,8\end{array}$ & $\begin{array}{c}3,5 \\
\pm 0,7 \\
\end{array}$ \\
\hline
\end{tabular}

$\mathrm{a}, \mathrm{b}$ - Letras minúsculas diferentes entre os momentos dentro de cada grupo, indicam diferenças significativas $(\mathrm{P}<0,05)$. Momentos: M0 - basal; M1 - 12 horas; M2 - 24 horas; M3 - 36 horas; M4 - 48 horas; M5 - 60 horas.

Fonte: Elaboração dos autores. 
Tabela 3. Valores médios e desvio padrão dos valores obtidos pelo eritrograma (eritrócitos, hemoglobina, hematócrito) e leucograma (leucócitos, neutrófilos, linfócitos, monócitos, eosinófilos, basófilos e bastonetes) nos grupos RL e HES, nos momentos $\mathrm{M}_{0}, \mathrm{M}_{1}, \mathrm{M}_{2}$ e $\mathrm{M}_{3}$.

\begin{tabular}{|c|c|c|c|c|c|}
\hline Parâmetros & Grupos & $\mathbf{M}_{0}$ & $\mathbf{M}_{1}$ & $\mathbf{M}_{2}$ & $\mathbf{M}_{3}$ \\
\hline \multirow{3}{*}{ Eritrócitos (x10/ul) } & HES & $\begin{array}{c}6,1 \\
\pm 1,2\end{array}$ & $\begin{array}{c}4,8 \\
\pm 0,9\end{array}$ & $\begin{array}{c}4,8 \\
\pm 0,8\end{array}$ & $\begin{array}{c}4,9 \\
\pm 0,9\end{array}$ \\
\hline & RL & $\begin{array}{c}6,0 \\
\pm 1,8\end{array}$ & $\begin{array}{c}5,1 \\
\pm 1,3\end{array}$ & $\begin{array}{c}4,6 \\
\pm 1,2\end{array}$ & $\begin{array}{c}4,7 \\
\pm 1,2\end{array}$ \\
\hline & HES & $\begin{array}{c}13,3 \\
\pm 3,5 \mathrm{a}\end{array}$ & $\begin{array}{c}10,4 \\
\pm 2,2 \mathrm{~b}\end{array}$ & $\begin{array}{c}9,4 \\
\pm 1,5 \mathrm{~b}\end{array}$ & $\begin{array}{l}10,6 \\
\pm 1,9\end{array}$ \\
\hline Hemoglobina (g/dl) & RL & $\begin{array}{c}13,3 \\
\pm 3,9 \mathrm{a}\end{array}$ & $\begin{array}{l}10,8 \\
\pm 2,8\end{array}$ & $\begin{array}{c}10,8 \\
\pm 3,0 \mathrm{~b}\end{array}$ & $\begin{array}{c}9,6 \\
\pm 0,8\end{array}$ \\
\hline \multirow{3}{*}{ Hematócrito (\%) } & HES & $\begin{array}{l}39,5 \\
\pm 9,0\end{array}$ & $\begin{array}{l}32,0 \\
\pm 7,6\end{array}$ & $\begin{array}{l}29,7 \\
\pm 5,0\end{array}$ & $\begin{array}{l}33,0 \\
\pm 4,5\end{array}$ \\
\hline & RL & $\begin{array}{c}41,4 \\
\pm 12,4 \mathrm{a}\end{array}$ & $\begin{array}{c}34,0 \\
\pm 8,2 \mathrm{~b}\end{array}$ & $\begin{array}{c}32,6 \\
\pm 8,9 \mathrm{~b}\end{array}$ & $\begin{array}{l}29,5 \\
\pm 6,3\end{array}$ \\
\hline & HES & $\begin{array}{c}3400 \\
\pm 2891\end{array}$ & $\begin{array}{c}3922 \\
\pm 3764\end{array}$ & $\begin{array}{c}5193 \\
\pm 4775\end{array}$ & $\begin{array}{c}5836 \\
\pm 3236\end{array}$ \\
\hline Leucócitos $(/ u \mathrm{~L})$ & RL & $\begin{array}{c}4433 \\
\pm 6020\end{array}$ & $\begin{array}{c}4760 \\
\pm 6649\end{array}$ & $\begin{array}{l}2770 \\
\pm 471\end{array}$ & $\begin{array}{l}14375 \\
\pm 2227\end{array}$ \\
\hline \multirow{3}{*}{ Neutrófilos $(/ u \mathrm{~L})$} & HES & $\begin{array}{c}1409 \\
\pm 1419\end{array}$ & $\begin{array}{c}1119 \\
\pm 1286\end{array}$ & $\begin{array}{c}3778 \\
\pm 3877\end{array}$ & $\begin{array}{c}2796 \\
\pm 1673\end{array}$ \\
\hline & RL & $\begin{array}{c}2213 \\
\pm 2496\end{array}$ & $\begin{array}{c}1646 \\
\pm 2759\end{array}$ & $\begin{array}{c}566 \\
\pm 466,6\end{array}$ & $\begin{array}{r}10617 \\
\pm 171,8\end{array}$ \\
\hline & HES & $\begin{array}{c}1509 \\
\pm 1819\end{array}$ & $\begin{array}{c}2077 \\
\pm 1555\end{array}$ & $\begin{array}{l}1390 \\
\pm 843\end{array}$ & $\begin{array}{c}1690 \\
\pm 1041\end{array}$ \\
\hline Linfócitos $(/ u \mathrm{~L})$ & RL & $\begin{array}{c}883 \\
\pm 557\end{array}$ & $\begin{array}{l}2034 \\
\pm 861\end{array}$ & $\begin{array}{c}1779 \\
\pm 1121\end{array}$ & $\begin{array}{c}2373 \\
\pm 2450\end{array}$ \\
\hline \multirow{3}{*}{ Monócitos $(/ u \mathrm{~L})$} & HES & $\begin{array}{c}625 \\
\pm 616\end{array}$ & $\begin{array}{c}964 \\
\pm 1040\end{array}$ & $\begin{array}{c}874 \\
\pm 440\end{array}$ & $\begin{array}{c}961 \\
\pm 879\end{array}$ \\
\hline & RL & $\begin{array}{c}1756 \\
\pm 3198\end{array}$ & $\begin{array}{c}1218 \\
\pm 1938\end{array}$ & $\begin{array}{c}341 \\
\pm 244\end{array}$ & $\begin{array}{l}1020 \\
\pm 548\end{array}$ \\
\hline & HES & $\begin{array}{c}122 \\
\pm 103\end{array}$ & $\begin{array}{c}249 \\
\pm 269\end{array}$ & $\begin{array}{c}225 \\
\pm 396\end{array}$ & $\begin{array}{c}126 \\
\pm 112\end{array}$ \\
\hline Eosinófilos $(/ u \mathrm{~L})$ & RL & $\begin{array}{c}450 \\
\pm 901\end{array}$ & $\begin{array}{c}935 \\
\pm 1796\end{array}$ & $\begin{array}{c}109 \\
\pm 89,7\end{array}$ & $\begin{array}{l}921 \\
\pm 32\end{array}$ \\
\hline \multirow[b]{2}{*}{ Basófilos $(/ u \mathrm{~L})$} & HES & 0 & 0 & 0 & 0 \\
\hline & RL & $\begin{array}{c}25 \\
\pm 21\end{array}$ & 0 & 0 & 0 \\
\hline \multirow[b]{2}{*}{ Bastonetes $(/ u \mathrm{~L})$} & HES & $\begin{array}{c}162 \\
\pm 122\end{array}$ & $\begin{array}{c}91 \\
\pm 54\end{array}$ & $\begin{array}{l}125 \\
\pm 68\end{array}$ & $\begin{array}{c}291 \\
\pm 178\end{array}$ \\
\hline & $\mathbf{R L}$ & $\begin{array}{c}194 \\
\pm 147\end{array}$ & $\begin{array}{c}33 \\
\pm 32\end{array}$ & $\begin{array}{c}99 \\
\pm 54\end{array}$ & $\begin{array}{l}286 \\
\pm 42\end{array}$ \\
\hline
\end{tabular}

$\mathrm{a}, \mathrm{b}$ - Letras minúsculas diferentes entre os momentos dentro de cada grupo, indicam diferenças significativas $(\mathrm{P}<0,05)$. Momentos: M0 - basal; M1 - 12 horas; M2 - 24 horas; M3 - 36 horas.

Fonte: Elaboração dos autores. 
Os animais do HES tiveram uma porcentagem de desidratação significativamente menor nos tempos que sucederam o basal $\left(\mathrm{M}_{0}\right)$ (momento em que os animais eram internados) conforme Tabela 1, ou seja, o tratamento com HES 130/0,4 foi eficaz em reduzir o grau de desidratação nos animais. No entanto, não houve diferença significativa em comparação com o grupo RL. Segundo Mazzafero (2008) a carga negativa dos colóides, atrai o sódio, e o seu peso molecular, o qual impede a saída destes dos vasos, favorecendo a retenção de água por um período de 2 a 36 horas, permitindo conservar a pressão oncótica intravascular e diminuir a quantidade de fluidos necessária para restabelecer a volemia. Já os cristalóides, conforme Prough et al. (2009) tem como maior desvantagem o aumento transitório do volume intravascular, necessitando assim de maior quantidade de infusão, além da possibilidade de ocorrer edema periférico e pulmonar.

No grupo RL, 70\% dos animais apresentaram anorexia até o $\mathrm{M}_{5}$ e no HES 77,7\%; sendo que no HES, 44,4\% apresentaram vômito e no RL, 50\%. De acordo com Kalli et al. (2010), os sinais clínicos de letargia ou depressão, vômito, linfopenia e hipoalbuminemia não tiveram relação com o tempo de internamento dos pacientes

A porcentagem de animais com hematoquezia foi de $25,9 \%$ no HES e $15 \%$ no RL, sendo que apenas um animal do HES permaneceu com hematoquezia até $\mathrm{M}_{5}$, os demais cessaram após início do tratamento. Somente um animal chegou apresentando melena, o qual foi alocado aleatoriamente no grupo HES, cessando após 24 horas da instituição do tratamento. Quanto à presença de diarréia, no HES $37,0 \%$ dos pacientes apresentaram e no RL, 45\%, entretanto apenas dois animais (um do grupo HES e outro do grupo RL) permaneceram com diarréia até $\mathrm{M}_{4}$, os demais interromperam após 24 horas de internamento.

A alta taxa de anorexia, vômito e diarréia encontrada neste estudo, está de acordo com os dados descritos por Lamm e Rezabek (2008). De acordo com Hoskins (2004), o aporte oral de alimentos e de líquidos deve ser interrompido até que o vômito cesse por pelo menos 24 horas. Quando a êmese estiver sob controle, pequenas quantidades de dieta branda são oferecidas inicialmente, retornandose gradualmente para a dieta normal. O vomito frequênte ou persistente causado pelo parvovírus pode ser manejado com metoclopramida $(0,5 \mathrm{mg} /$ $\mathrm{kg}$; SC; TID ou por infusão continua de 1 a $2 \mathrm{mg} /$ $\mathrm{kg}$ por 24 horas, diluída em fluidos intravenosos), o que corrobora com a metodologia utilizada neste estudo.

No presente estudo não foram verificados distúrbios ácido-base, fato que pode estar relacionado ao reduzido número de animais utilizado. Jacobs, Weiser e Hall (2007) demonstrou redução da concentração de $\mathrm{CO}_{2}$ total em menos de $17 \%$ de um total de 134 cães com enterite por parvovírus. Heald, Jones e Schmidt (2007) revelou que a alcalose metabólica e a hipocloremia foram mais frequentes que a acidose metabólica em cães com parvovirose. De acordo com a Tabela 2, no grupo RL, observou-se aumento da $\mathrm{PaO}_{2}$ nos momentos $\mathrm{M}_{1}, \mathrm{M}_{2}$ e $\mathrm{M}_{4}$, quando comparado ao momento de internação, devido ao fato de que segundo Kirby e Rudloff(2004) o oxigênio está ligado à hemoglobina intravascular nos pulmões para ser transportado para os tecidos periféricos, e isto depende de um volume adequado de líquido para conduzir o oxigênio para os tecidos, contribuindo para uma melhor perfusão, o que justifica o menor valor de $\mathrm{PaO}_{2}$ observado em $\mathrm{M}_{0}$, pois os animais chegavam desidratados.

Observou-se diminuição nos valores de potássio apenas no HES ( $\mathrm{M}_{4}$ e $\left.\mathrm{M}_{5}\right)$ em relação a M0. Filhotes com enterite por CPV são propensos a desenvolver hipocalemia e hipoglicemia (especificamente raças miniaturas), devido à anorexia em curso, vômitos e diarréia. Hipocalemia severa pode resultar em fraqueza muscular profunda, arritmias cardíacas e poliúria (PRITTIE, 2004). Segundo Nappert et al. (2007), num estudo com 22 cães, naturalmente infectados por parvovirus, verificou-se baixas concentrações plasmáticas de potássio quando 
comparados com o grupo controle, o qual era composto por animais saudáveis.

Houve redução na concentração de fósforo, no HES, nos momentos $M_{1}$ até $M_{5}$, e no RL em $M_{1}, M_{3}$ e $M_{4}$ em relação ao $M_{0}$. Segundo Dibartola (2007) isso pode ser explicado pela presença de vômito, má absorção, deficiência na dieta ou menor ingestão; fato observado nos pacientes de ambos os grupos. Porém, as diminuições observadas em ambos os grupos não ultrapassaram o limite inferior para a espécie canina, não caracterizando hipofosfatemia.

No grupo RL, houve diminuição nos níveis de magnésio no decorrer do internamento; o que segundo Bateman (2007) é devido à anorexia e ao tempo de restrição alimentar a que eram submetidos, devido à presença de vômito. Pacientes veterinários com diarréia raramente apresentam hipomagnesemia e são verificados com mais frequência em animais com enteropatia ocasionada por perda de proteína (KIMMEL; WADDEL; MICHEL, 2007).

Os dados obtidos pelo leucograma não apresentaram diferença significativa dentro dos grupos, sendo que os valores dos leucócitos se mantiveram baixos, havendo pequena elevação no decorrer do tratamento, conforme demonstra a Tabela 3. A contagem de leucócitos durante enterite por CPV é geralmente caracterizada como significativamente deprimida, com uma linfopenia transitória. Segundo Goddard et al. 2008, a monocitopenia inicial (pré tratamento) foi associada com um resultado negativo, enquanto a contagem de leucócitos (>4500/uL); linfócitos (>1000/ uL); monócitos $(>150 / \mathrm{uL}) ;$ e eosinófilos $(>100 / \mathrm{uL})$, bem como o desvio à esquerda, aparecendo nas primeiras $24 \mathrm{~h}$ após a admissão, tem um alto valor preditivo positivo para os cães sobreviventes ao parvovírus. Esses dados apoiam o fato de que as contagens sequenciais de leucócitos durante o tempo de internamento parecem mais confiáveis para a previsão de resultado em comparação com o número de leucócitos no momento da admissão.
Ainda de acordo com a Tabela 3, a hemoglobina no HES foi menor em $\mathrm{M}_{2}$ quando comparado ao $\mathrm{M}_{0}$, e os níveis de hematócrito mantiveram-se sem diferença significativa, porém no RL os valores de hematócrito e hemoglobina foram menores em $M_{1}$ e $M_{2}$, quando comparado a $M_{0}$. A anemia é um achado hematológico comum em enterite por CPV, especialmente nas fases posteriores à doença grave. A causa desta é pouco provável que seja a supressão da eritropoiese, pois os glóbulos vermelhos têm uma meia-vida longa para o curto período durante o qual o vírus suprime a produção. O hematócrito reduzido é mais provável que seja o resultado de uma combinação de hemorragia intestinal e terapia de reidratação (HOSKINS, 1997).

\section{Conclusão}

Ambos os tratamentos foram efetivos na reposição volêmica dos cães com parvovirose, não diferindo em nenhuma variável analisada. Estudos adicionais são necessários para avaliar os benefícios do hidroxietilamido 130/0,4 em cães com gastroenterite hemorrágica por parvovírus.

\section{Comitê de Ética e Biossegurança}

Este estudo foi aprovado pelo Comitê de Ética e Bem Estar Animal (CETEA) da instituição de origem (Protocolo: 11/2010).

\section{Referências}

BATEMAN, S. Distúrbios relacionados ao magnésio: déficil e excesso. In: DIBARTOLA, S. P. Anormalidades de fluidos, eletrólitos e equilíbrio ácido-basico. 3. ed. São Paulo: Roca, 2007. cap. 8, p. 212-229.

DIBARTOLA, S. P. Anormalidades de fluidos, eletrólitos e equilíbrio ácido-básico na clínica de pequenos animais. 3. ed. São Paulo: Roca, 2007. 664 p.

ESFANDIARI, J.; KLINGEBORN, B. A comparative study of a new rapid and one-step test for the detection of parvovirus in faeces form dogs, cats and mink. Journal of Veterinary Medicine, Wiley Online Library, Malden, v. 47, n. 2, p. 145-153, 2000. 
GODDARD, A.; LEISEWITZ, A. L.; CHRISTOPHER, M. M.; DUNCAN, N. M; BECKER, P. L. Prognostic usefulness of blood leukocyte changes in canine parvoviral enteritis. Journal of Veterinary Internal Medicine, Wiley Online Library, Malden, v. 22, n. 2, p. 309-316, 2008.

HEALD, R. D.; JONES, B. D.; SCHMIDT, D. A. Blood gas and electrolyte concentrations in canine parvoviral enteritis in: DIBARTOLA, S. P. Anormalidades de fluidos, eletrólitos e equilíbrio ácido-basico. 3. ed. São Paulo: Roca, 2007. cap. 8, p. 212-229.

HOSKINS, J. D. Update on canine parvoviral enteritis. Veterinary Medicine, Barcelona, v. 92, n. 8, p. 694-709, 1997.

JACOBS, R. M.; WEISER, M. G.; HALL, R. L. Clinicopathologic findings of canine parvoviral enteritis. In: DIBARTOLA, S. P. Anormalidades de fluidos, eletrólitos e equilíbrio ácido-basico. 3. ed. São Paulo: Roca, 2007. cap. 8, p. 212-229.

KALLI, I.; LEONTIDES, L. S.; MYLONAKIS, M. E.; ADAMAMA-MORAITOU, K.; RALLIS, T.; KOUTINAS, A. F. Factors affecting the ocurrence, duration of hospitalization and final outcome in canine parvovirus infection. Research in Veterinary Science, London, v. 89, n. 2, p. 174-178, 2010.

KIMMEL, S. E.; WADDEL, L. S.; MICHEL, K. E. Hypomagnesemia and hypocalcemia associated with protein-losing enterophaty in Yorkshire terrier. In: DIBARTOLA, S. P. Anormalidades de fluidos, eletrólitos e equilíbrio ácido-basico. 3. ed. São Paulo: Roca, 2007. cap. 6, p. 120-194.

KIRBY, R.; RUDLOFF, E. Terapia de líquidos e eletrólitos. In: ETTINGER, S. J.; FELDMAN, E. C. Tratado de medicina interna veterinária. 5 . ed. Guanabara Koogan: São Paulo, 2004. cap. 78, p. 342-365.

LAMM, C. G.; REZABEK, G. B. Parvovirus infection in domestic companion animals. The Veterinary Clinics of North America. Small Animal Practice, Australia, v. 38, n. 4, p. 837-850, 2008.

LV, R.; ZHOU, Z. Q.; WU, H. W.; JIN, Y.; ZHOU, W.; XU, J. G. Hydroxyethyl starch exhibits anti-inflammatory effects in the intestines of endotoxemic rats. Anesthesia \& Analgesia, California, v. 103, n. 1, p. 149-55, 2006.
MARX, G.; PEDDER, S.; SMITH, L.; SWARAJ, S.; GRIME, S.; STOCKDALE, H.; LEUWER, M. Attenuation of capillary leakage by hydroxyethyl starch $(130 / 0.42)$ in a porcine model of septic shock. Critical Care Medicine, New Jersey, v. 34, n. 12, p. 3005-3010, 2006.

MAZZAFERO, E. M. Complications of fluid therapy. Veterinary Clinics of North America: Small Animal Practice, Australia, v. 38, n. 3, p. 607-619, 2008.

McCAW, D. L.; HOSKINS, J. D. Canine viral enteritis. In: GREENE, C. E. (Ed.). Infectious diseases of the dog and cat. Saunders Elsevier, St. Louis, 2006. p. 63-73.

NAPPERT, G.; DUNPHY, E.; RUBEN, D.; MANN, F. A. Determination of serum organic acids in puppies with naturally acquired parvoviral enteritis. In: DIBARTOLA, S. P. Anormalidades de fluidos, eletrólitos e equilíbrio ácido-basico. 3. ed. São Paulo: Roca, 2007. p. 253-286.

OTTO, C. M.; JACKSON, C. B.; ROGELL, E. J.; PRIOR, R. B.; AMMONS, W. S. Recombinant bactericidal/ permeability-increasing protein (rBPI21) for treatment of parvovirus enteritis: a randomized, double-blinded, placebo-controlled trial. Journal of Veterinary Internal Medicine, Wiley Online Library, Malden, v. 15, n. 4, p. 355-360, 2001.

PRITTIE, J. Canine parvoviral enteritis: a review of diagnosis, management, and prevention. Journal Veterinary Emergency Critical Care, v. 14, n. 3, p. 167$176,2004$.

PROUGH, D. S.; FUNSTON, J. S.; SVENSÉN, C. H.; WOLF, S. W. Fluids, electrolytes, and acidbase physiology. In: BARASH, P. G.; CULLEN, B. F.; STOELTING, R. K.; CAHALAN, M. K.; STOCK, M. C.; editors. Clinical anesthesia. 6. ed. Philadelphia: Lippincott Williams \&Wilkins, 2009. p. 291-326.

SAVIGNY, M.; MACINTIRE, D. K. Canine parvoviral enteritis. Standards of care: emergency and critical care medicine, Phillips Boulevard, Trenton, v. 9, n. 11, p. 1-6, 2007.

SHERDING, R. G. Vírus intestinais. In: BIRCHARD, S. J.; SHERDING, R. G. Manual saunders: clínica de pequenos animais. 2. ed. São Paulo: Roca, 2003. cap. 12, p. 121-129.

WALTZINGER, J.; BEPPERLING, F.; PABST, G. Effect of new HES specification $(6 \%$ HES $130 / 0,4)$ on blood and plasma volumes after bleeding in 12 healthy male volunteers. Clinical Drug Investigation, New Zealand, v. 17, n. 2, p. 119-25, 1999. 
